\title{
Discrete symmetries studies at KLOE-2
}

\author{
Daria Kisielewska $^{1, *}$ \\ for the KLOE-2 Collaboration \\ ${ }^{1}$ Institute of Physics, Jagiellonian University, Cracow, Poland
}

\begin{abstract}
The KLOE and KLOE-2 experiments at the Laboratori Nazionali di Frascati (Italy) collected almost $8 \mathrm{fb}^{-1}$ of integrated luminosity at the energy equal to the mass of $\phi$ meson. The excellent time resolution of the electromagnetic calorimeter and the very good accuracy on both momentum and vertex reconstruction of the tracking system allow to study discrete symmetries to the utmost precision as well as light meson spectroscopy, dark forces searches, hadronic cross-section measurements and studies of $\gamma \gamma$-physics. CPT symmetry test with the lepton charge asymmetry measured in $K_{S}$ semileptonic decays with $1.7 \mathrm{fb}^{-1}$ of KLOE data, tests of time reversal and CPT in transitions in $\phi \rightarrow K_{S} K_{L} \rightarrow \pi e v, 3 \pi^{0}\left(2 \pi^{0}\right)$ decays with newly acquired data with the KLOE-2 detector will be presented and discussed.
\end{abstract}

\section{Introduction}

The K LOng Experiment (KLOE) is located at electron-positron collider DAФNE in Frascati near Rome. The accelerator operates at the $\phi$ meson mass peak $\left(\sqrt{s}=m_{\phi} \approx 1019 \mathrm{MeV}\right)$. Decays of produced $\phi$ meson were registered by the KLOE experiment from 2001 to 2006. The data taking campaign has been restarted in 2014 and till march 2018 the KLOE-2 detector collected an additional dataset with an integrated luminosity of $5.5 \mathrm{fb}^{-1}$.

The experimental setup common to KLOE and KLOE-2 consists of cylindrical drift chamber (DC) and electromagnetic calorimeter (EMC). Both detectors are surrounding the $e^{+} e^{-}$interaction point and are embedded into 0.52 T magnetic field. The DC is filled with mixture of helium (90\%) and isobutane (10\%) and provides a vertex resolution of the order of $1 \mathrm{~mm}$ and transverse momentum accuracy better than $0.4 \%$ [1]. The EMC, made of lead and scintillating fibers, has energy and time resolution of $\sigma(E) / E=5.7 \% / \sqrt{E[\mathrm{GeV}]}$, and $\sigma_{t}=54 \mathrm{ps} / \sqrt{E[\mathrm{GeV}]} \oplus 140 \mathrm{ps}$, respectively, for photons and electrons [2].

The KLOE-2 detector is equipped with a set of new detectors [3]: (i) the Inner Tracker based on 4 layers of Cylindrical Gas Electron Multiplier detector aiming at improving tracking and vertexing resolution close to the interaction point, (ii) crystal (CCALT) [4] and tile (QCALT) [5] calorimeters covering the low polar angles and improving the detection of the photons that are coming from $K_{L}$ decays in the drift chamber, respectively, (iii) the two pairs of small angle tagging devices that allows to detect the low (Low Energy Tagger [6]) and high (High Energy Tagger [7]) energy $e^{+} e^{-}$originated from $e^{+} e^{-} \rightarrow e^{+} e^{-} X$ reactions.

\footnotetext{
*e-mail: daria.kisielewska@uj.edu.pl
} 


\section{CPT symmetry test with semileptonic decays}

One of possible tests of CPT symmetry is preformed by comparing the value of a lepton charge asymmetry for long- and short-lived kaons:

$$
A_{S / L}=\frac{\Gamma\left(K_{S / L} \rightarrow \pi^{-} e^{+} v\right)-\Gamma\left(K_{S / L} \rightarrow \pi^{+} e^{-} \bar{v}\right)}{\Gamma\left(K_{S / L} \rightarrow \pi^{-} e^{+} v\right)+\Gamma\left(K_{S / L} \rightarrow \pi^{+} e^{-} \bar{v}\right)}=2\left[\operatorname{Re}(\epsilon) \pm \operatorname{Re}(\delta)-\operatorname{Re}(y) \pm \operatorname{Re}\left(x_{-}\right)\right],
$$

where $\operatorname{Re}(\epsilon)$ and $\operatorname{Re}(\delta)$ are implying T and CPT violation in the $K^{0}-\bar{K}^{0}$ mixing, respectively. The $\operatorname{Re}(y)$ and $\operatorname{Re}\left(x_{-}\right)$parametrize the CPT violation in $K_{S} \rightarrow \pi e v$ decay amplitudes [8]. If there is no CPT symmetry violation, then the aforementioned asymmetries are expected to be identical: $A_{S}=A_{L}=2 \operatorname{Re}(\epsilon)$.

The charge asymmetry for $K_{L}$ was precisely determined from the $\mathrm{KTeV}$ experiment at Fermilab $A_{L}=\left(3.322 \pm 0.058_{\text {stat }} \pm 0.047_{\text {syst }}\right) \times 10^{-3}$ [9], while the most precise measurement of $A_{S}$ was conducted by the KLOE collaboration using $410 \mathrm{pb}^{-1}$ of integrated luminosity collected in 2001-2002: $A_{S}=\left(1.5 \pm 9.6_{\text {stat }} \pm 2.9_{\text {syst }}\right) \times 10^{-3}$ [10]. Recently, the KLOE-2 group reported a new measurement based on a four times larger data sample, corresponding to an integrated luminosity of $1.63 \mathrm{fb}^{-1}$ collected in 2004-2005 [11]: $A_{S}=\left(-4.9 \pm 5.7_{\text {stat }} \pm 2.6_{\text {syst }}\right) \times$ $10^{-3}$, which improves the statistical accuracy of previous determination by almost a factor two. Taking into account the correlation between both measurements, their combination is:

$$
A_{S}=\left(-3.8 \pm 5.0_{\text {stat }} \pm 2.6_{\text {syst }}\right) \times 10^{-3} \text {. }
$$

This value, combine with $\mathrm{KTeV}$ result on $A_{L}$ and providing the $\operatorname{Re}(\delta)$ [12] and $\operatorname{Re}(\epsilon)$ [13], allows to extract the CPT violating parameters [11]:

$$
\begin{gathered}
\operatorname{Re}\left(x_{-}\right)=(-2.0 \pm 1.4) \times 10^{-3}, \\
\operatorname{Re}(y)=(1.7 \pm 1.4) \times 10^{-3},
\end{gathered}
$$

which are consistent with CPT invariance and improve by almost a factor of two the previous results [10].

With KLOE-2 dataset, the rare $K_{S}$ decays are currently being studied: with $5.5 \mathrm{fb}^{-1}$ total integrated luminosity a pure data sample of about $5 \times 10^{9}$ tagged $K_{S}$ decays will be available. In addition, the event reconstruction will be improved thanks to the new Inner Tracker detector aiming at improving the tracking and vertexing resolution close to the interaction point.

\section{Tests of Time reversal and CPT in transitions in $\phi \rightarrow K_{S} K_{L} \rightarrow \pi e v, 3 \pi^{0}\left(2 \pi^{0}\right)$ decays}

The gathered data sample allows for direct tests of discrete symmetries in neutral kaons transitions $[14,15]$. Studies are based on comparison between rates of processes and its timereversal conjugates, obtained by an exchange of initial and final states. An example of this analysis is the study of the kaon transitions between their pure flavour $\left\{K^{0}, \bar{K}^{0}\right\}$ and CPdefinite $\left\{K_{+}, K_{-}\right\}$states. The former are identified by semileptonic decays $K^{0} \rightarrow \pi^{-} e^{+} v$ and $\bar{K}^{0} \rightarrow \pi^{+} e^{-} \bar{v}$ whereas the latter must decay hadronically $K_{+} \rightarrow \pi^{+} \pi^{-}$and $K_{-} \rightarrow 3 \pi^{0}$.

The KLOE-2 is presently pursuing the measurement of the ratios:

$$
\begin{aligned}
& R_{2}(\Delta t)=\frac{P\left[K^{0}(0) \rightarrow K_{-}(\Delta t)\right]}{P\left[K_{-}(0) \rightarrow K_{0}(\Delta t)\right]}=\frac{I\left(l^{-}, 3 \pi^{0} ; \Delta t\right)}{I\left(\pi \pi, l^{+} ; \Delta t\right)} \times \frac{1}{C}, \\
& R_{4}(\Delta t)=\frac{P\left[\bar{K}^{0}(0) \rightarrow K_{-}(\Delta t)\right]}{P\left[K_{-}(0) \rightarrow \bar{K}_{0}(\Delta t)\right]}=\frac{I\left(l^{+}, 3 \pi^{0} ; \Delta t\right)}{I\left(\pi \pi, l^{-} ; \Delta t\right)} \times \frac{1}{C},
\end{aligned}
$$


where constant $C=\frac{B R\left(K_{L} \rightarrow 3 p i^{0}\right) \cdot \Gamma_{L}}{B R\left(K_{S} \rightarrow \pi \pi\right) \cdot \Gamma_{S}}$ involves well determined kaon parameters. The asymptotic behaviour of these ratios for large time intervals $\Delta t$ is related to time-reversal violating parameter $\epsilon$ as:

$$
R_{2,4}\left(\Delta t \gg \tau_{S}\right) \simeq 1 \mp 4 \operatorname{Re}(\epsilon) .
$$

The analysis flow is now tested using the data gathered by KLOE detector, and will be applied to the KLOE-2 dataset. The Figure 1 presents preliminary distributions of $R_{2}$ and $R_{4}$ rates.
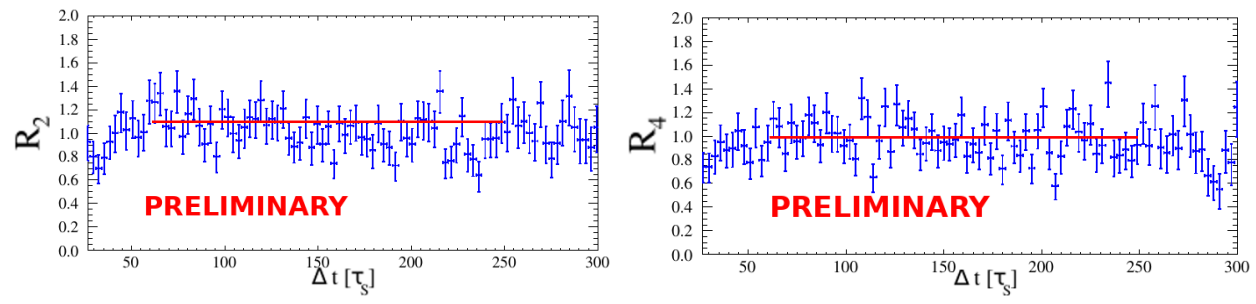

Figure 1. Preliminary distribution of the transition probability ratios $R_{2}$ (left) and $R_{4}$ (right) as a function of a proper decay time difference $\Delta t$. Points are the KLOE data, while the red line is a fitted line to data points.

The observables sensitive for CPT symmetry violation are constructed in similar manner [15]: $R_{2,4}^{C P T} \sim I\left(l^{\mp}, 3 \pi^{0} ; \Delta t\right) / I\left(\pi \pi, l^{\mp} ; \Delta t\right)$. The ratio of the aforementioned CPT-violating ratios, in the asymptotic region of $\Delta t \gg \tau_{S}$, is related to the CPT-violating parameters in the following way:

$$
R_{2}^{C P T} / R_{4}^{C P T}=1-8 \operatorname{Re}(\delta)-8 \operatorname{Re}\left(x_{-}\right) \approx 1+2\left(A_{L}-A_{S}\right) .
$$

Figure 2 presents a preliminary result obtained with the KLOE dataset.

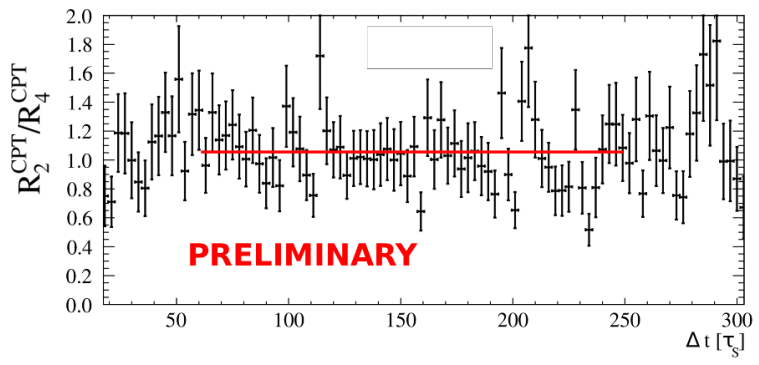

Figure 2. Preliminary distribution of the CPT-asymmetric double ratio obtained with the KLOE data. Points are the KLOE data, while the red line is a fitted line to data points.

\section{Summary}

The KLOE dataset is still exploited to provide more precise results as in the case of lepton charge asymmetry. The KLOE-2 provides not only larger statistics of $5.5 \mathrm{fb}^{-1}$ but also improved event reconstruction due to new detector components. The analysis of KLOE-2 data 
allows to make a significant progress in determining the $A_{S}$ value and searching for CP violation in $K_{S} \rightarrow 3 \pi^{0}$ decay. Moreover, collected data sample allows to preform, for the first time, the statistically significant test of discrete symmetries in transition.

We warmly thank our former KLOE colleagues for the access to the data collected during the KLOE data taking campaign. We thank the DAФNE team for their efforts in maintaining low background running conditions and their collaboration during all data taking. We want to thank our technical staff: G.F. Fortugno and F. Sborzacchi for their dedication in ensuring efficient operation of the KLOE computing facilities; M. Anelli for his continuous attention to the gas system and detector safety; A. Balla, M. Gatta, G. Corradi and G. Papalino for electronics maintenance; C. Piscitelli for his help during major maintenance periods. This work was supported in part by the Polish National Science Centre through the Grants No. 2013/08/M/ST2/00323, 2013/11/B/ST2/04245, 2014/14/E/ST2/00262, 2014/12/S/ST2/00459, 2016/21/N/ST2/01727, 2016/23/N/ST2/01293, 2017/26/M/ST2/00697.

\section{References}

[1] M. Adinolfi et al., Nucl. Instrum. Meth. A 488, 51 (2002)

[2] M. Adinolfi et al., Nucl. Instrum. Meth. A 482, 364 (2002)

[3] G. Amelino-Camelia et al., Eur. Phys. J. C 68, 619 (2010)

[4] M. Cordelli et al., Nucl. Instrum. Meth. A 718, 81 (2013)

[5] A. Balla et al., Nucl. Instrum. Meth. A 718, 95 (2013)

[6] D. Babusci et al., Nucl. Instrum. Meth. A 617, 81 (2010)

[7] D. Babusci et al., Nucl. Instrum. Meth. A 718, 577 (2013)

[8] L. Maiani, in The Second DAФNE Physics Handbook, edited by L. Maiani, G. Pancheri, N. Paver (INFN-LNF, Frascati, 1995), Vol. I, p. 3

[9] A. Alavi-Harati et al., Phys. Rev. Lett. 88, 181601 (2002)

[10] F. Ambrosino et al., Phys. Lett. B 636, 173 (2006)

[11] A. Anastasi et al., Accepted at: JHEP (2018), arXiv: 1806.08654

[12] C. Patrignani, et al. (Particle Data Group), Chin. Phys. C 40, 100001 (2016)

[13] F. Ambrosino et al., JHEP 12, 11 (2006)

[14] J. Bernabeu, A.D. Domenico, P. Villanueva-Perez, Nucl. Phys. B 868, 102 (2013)

[15] J. Bernabeu, A. Di Domenico, P. Villanueva-Perez, JHEP 10, 139 (2015) 